\title{
Lattice distortion and electron charge redistribution induced by defects in graphene
}

Wei Zhang, ${ }^{1,2}$ Wen-Cai Lu, ${ }^{1,3}$ Hong-Xing Zhang ${ }^{1}$, K. M. Ho ${ }^{2}$, and C. Z. Wang, ${ }^{2, *}$

${ }^{1}$ International Joint Research Laboratory of Nano-Micro Architecture Chemistry and Institute of Theoretical Chemistry, Jilin University, Changchun, Jilin 130023, People's Republic of China

${ }^{2}$ Ames Laboratory-U.S. DOE and Department of Physics and Astronomy, Iowa State University, Ames, Iowa 50011, USA

${ }^{3}$ Department of Physics and State Key Laboratory Cultivation Base of Advanced Fibers and Textile Materials, Qingdao University, Qingdao, Shandong 266071, People's Republic of China

\begin{abstract}
Lattice distortion and electronic charge localization induced by vacancy and embedded-atom defects in graphene were studied by tight-binding (TB) calculations using the recently developed three-center TB potential model. We showed that the formation energies of the defects are strongly correlated with the number of dangling bonds and number of embedded atoms, as well as the magnitude of the graphene lattice distortion induced by the defects. We also showed that the defects introduce
\end{abstract}


localized electronic states in the graphene which would affect the electron transport properties of graphene.

Keywords: Tight-binding calculation; Defects in graphene; Lattice distortion; Localized electronic states. 


\section{Introduction}

Graphene has appeared as one of the most remarkable new materials of the past 10 years due to its distinctive electronic, mechanical, and thermal properties [1-3]. However, mechanical strains induced by defects often give rise to surprising changes in the physical and chemical properties of graphene [4]. In recent years, novel physical properties in graphene induced by mechanical strain has been studied both experimentally [5-8] and theoretically [1,9-10]. Uniaxial strain can be induced in graphene by bending the substrates on which graphene is elongated [11]. Craco et al. unveil hidden electronic properties of isotropically strained graphene [12]. Jain et al. simulated buckling caused by strong long-range relaxations of typical defects such as the Stone-Wales (SW) defect in graphene [13]. The critical strain at which formation of SW defects in graphene becomes thermodynamically favorable was calculated to be $6-9 \%[14-16]$ and $12-17 \%[14,16]$, when the strain is applied in the zigzag and armchair directions, respectively. The geometry of stable dislocations also depends critically on whether they are formed from adatoms or vacancies $[17,18]$. Structure and deformation of the monovacancy near the edge of the graphene layer have been often observed by experiments $[19,20]$ and have been studied by theoretical calculations [21]. Gong et al. showed that bond rotations at the edge of graphene can help to reduce the overall strain in the system [22]. Chen et al. show that bond rotations reduce the maximum single bond strain reached within a divacancy (DV) and help distribute the strain over a larger number of bonds to minimize the peak magnitude [23].

Understanding how defects deform graphene helps build an accurate description 
of both elasticity and plasticity in graphene [24-26]. While many theoretical and experimental studies have been devoted to elucidating the Stone-Wales (SW) defect structures and their electronic properties, much less studies have provided systematic evidence to connect other vacancy and embedded-atom defect structures with their lattice distortion and local electronic properties. In this paper, we performed a systematic calculation to study the lattice distortion and electronic charge variation caused by various vacancy and embedded-atom defects in graphene, in order to gain more comprehensive and deeper understanding of the properties of graphene subjected to point defects.

\section{Calculation Methods}

The calculations are performed using the three-center tight-binding potential recently developed [27]. The interatomic interactions in TB method are based on quantum mechanics descriptions thus has better accuracy than empirical classical potential can offer. At the same time, it can provide faster computing speed in comparison with first-principles methods such as density functional theory (DFT) because it use minimal basis and the hopping and overlap integrals are parametrized. It has been demonstrated that the newly developed three-center TB potential for carbon exhibits excellent accuracy and transferability. It describes well the band structures, binding energies and other properties of various carbon crystalline structures and surfaces. It is also very efficient for large scale atomistic simulations and can accurately describe the structures and energies of various defects in graphene. Details of TB methods 
have been described in previous literatures [27]. The calculations presented in this paper were performed using a rectangular graphene supercell containing 1000 atoms and with a vacuum region of $50 \AA$ in the direction perpendicular to the graphene and with periodic boundary conditions. The defect structures are fully relaxed until the forces on every atom are less than $0.01 \mathrm{eV} / \AA$.

Our calculations are based on the most stable vacancy and adatom defects structures in graphene obtained from our calculations as well as those in the literature $[28,29]$. These structures are shown in Fig. 1. The simplest defects in graphene is the Stone Wales (SW) defect where four hexagons are transformed into two pentagons and two heptagons (i.e., 55-77 defect) by rotating one of the C-C bonds by $90^{\circ}$. Single vacancy (SV) in graphene prefers a five-membered and a nine-membered ring (i.e., a 5-9 defect). The stable structures of divacancy defect in graphene is the three-pentagons and three-heptagons $V_{2}(555-777)$ [28]. The lowest energy structure of trivacancy defect is composed of two five-membered and one ten-membered (heart-shape) rings, i.e., $\mathrm{V}_{3}$ (5-10-5) which has one dangling bond. The $\mathrm{V}_{4}(5555-66-7777)$ is the most favorable structure of tetravacancy defect which has six pentagons, six heptagons and two hexagons [29]. The stable structure of hexvacancy defect $\mathrm{V}_{6}$ is obtained by removing a hexagonal ring from the graphene. The most stable structure for embedding two atoms contains a pair of joined pentagonal carbon rings placed between a pair of heptagonal rings, i.e., a $A_{2}(7-55-7)$ structure. Finally, the lowest-energy structure for embedding four atoms consist a core of a pair of adjacent five-membered rings, a hexagonal ring and another five-membered ring, 
surrounded by three seven-membered rings. We call this defect a $\mathrm{A}_{4}(55-6-5-777)$

defect.

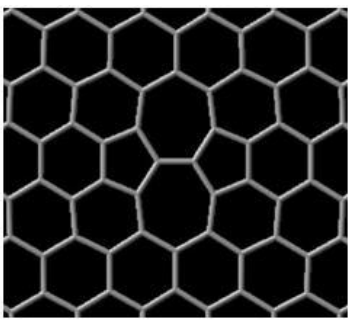

(a) (5.05)

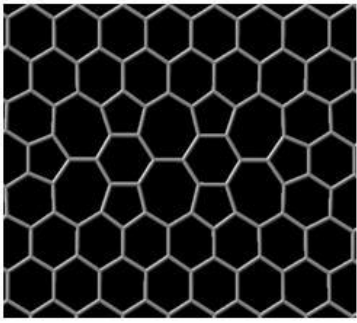

(e) $(\mathbf{1 1 . 0 0})$

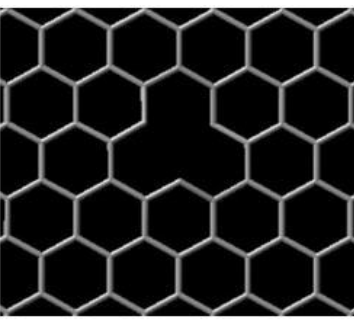

(b) (6.97)

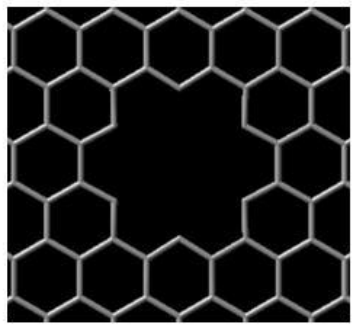

(f) $(\mathbf{1 2 . 0 2})$

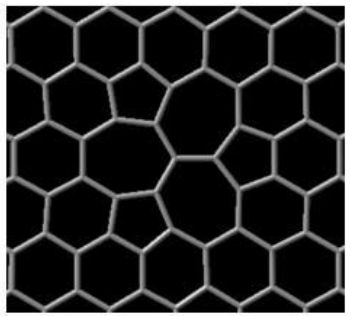

(c) $(6.88)$

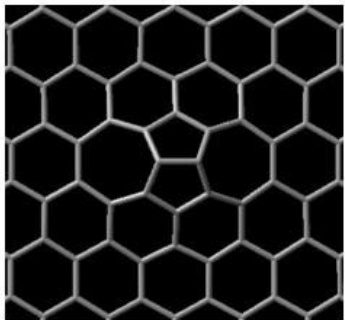

(g) $(6.31)$

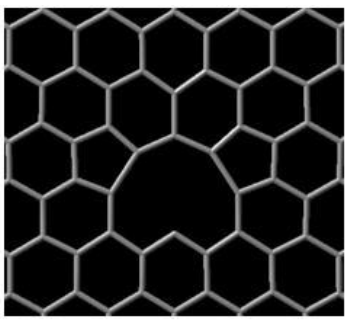

(d) $($ 10.33)

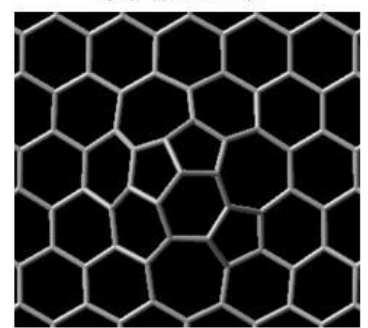

(h) $(8.79)$

Fig. 1 The lowest-energy structures and formation energies (in $\mathrm{eV}$ shown in the parenthesis) of (a) $\mathrm{SW}(55-77)$, (b) $\mathrm{SV}(5-9)$, (c) $\mathrm{V}_{2}$ (555-777), (d) $\mathrm{V}_{3}(5-10-5)$, (e) $\mathrm{V}_{4}(5555-66-7777)$, (f) $\mathrm{V}_{6}$, (g) $\mathrm{A}_{2}(7-55-7)$, and (h) $\mathrm{A}_{4}(55-6-5-777)$ defects calculated by the 3 -center TB.

\section{Results and Discussions}

\subsection{Lattice distortion induced by defects in graphene}

Defects in graphene can induce significant distortions to the graphene lattice. Such distortions can spread far away from the defects because most of the distortions are elastic in nature. Since TB calculation can handle a large number of atoms, it is very useful for studying the structure distortion caused by the defects in graphene. In Fig. 2, we show the bond length variation in the defect structures with respect to the 
equilibrium bond length $(1.42 \AA)$ in perfect graphene. Larger lattice distortions can be well seen at the vicinity of the defects, some bonds are stretched (brown or red) and some are compressed (blue or purple) which can also be seen clearly in Fig. 2. Away from the defects, the distortions become smaller but can extend to large distance from the center of the defect, particularly for $\mathrm{SW}$, and $\mathrm{V}_{2}-\mathrm{V}_{4}$ defects. For these defects, the bonds are found to be compressed in some directions (cyan) and stretched in other directions (light green). The lattice distortion in $\mathrm{SV}$ and $\mathrm{V}_{6}$ are more localized due to the formation of the dangling bonds around the vacancy. In addition to the in-plane distortion, the $\mathrm{SW}, \mathrm{A}_{2}$, and $\mathrm{A}_{4}$ defects also have significant vertical distortion as one can see from Fig. 2. In particular, the SW exhibits vertical cosine distortions with buckling heights of 2.10. The formation of the bulge along the z-direction in $\mathrm{A}_{2}$ and $\mathrm{A}_{4}$ structures also help to localize the strain around the defects.

We have also calculated the bond-angle distortions cause by the defects. Large bond-angle distortions occur when the hexagons in graphene change to other polygons. Angle distortions with the hexagons are relatively small. More details about the lattice distortions induced by the defects can also be found in Table 1 where the sum of square of deviation in bond length and relative bond angles are presented. We found that the defect energies can be well modeled using the information of bond-length and bond-angle distortions, as well as the number of dangling bonds and the number of embedded atoms. By expressing the defect energy as

$$
E_{d}=\alpha \sum_{m=1}^{N_{b}}\left(\Delta r_{m}\right)^{2}+\beta \sum_{n=1}^{N_{a}}\left(\frac{\Delta \theta_{n}}{\theta_{0}}\right)^{2}+\gamma N_{d}+\delta_{2} N_{A 2}+\delta_{4} N_{A 4}
$$

Where $\Delta r_{m}$ is the deviation of bond length from its equilibrium value in perfect 
graphene and $\mathrm{N}_{b}$ is the total number of nearest neighbor bonds; $\Delta \theta_{n}$ is deviation of bond angle from the perfect band-angle $\theta_{0}\left(=120^{\circ}\right)$ in graphene and $\mathrm{N}_{a}$ is the total

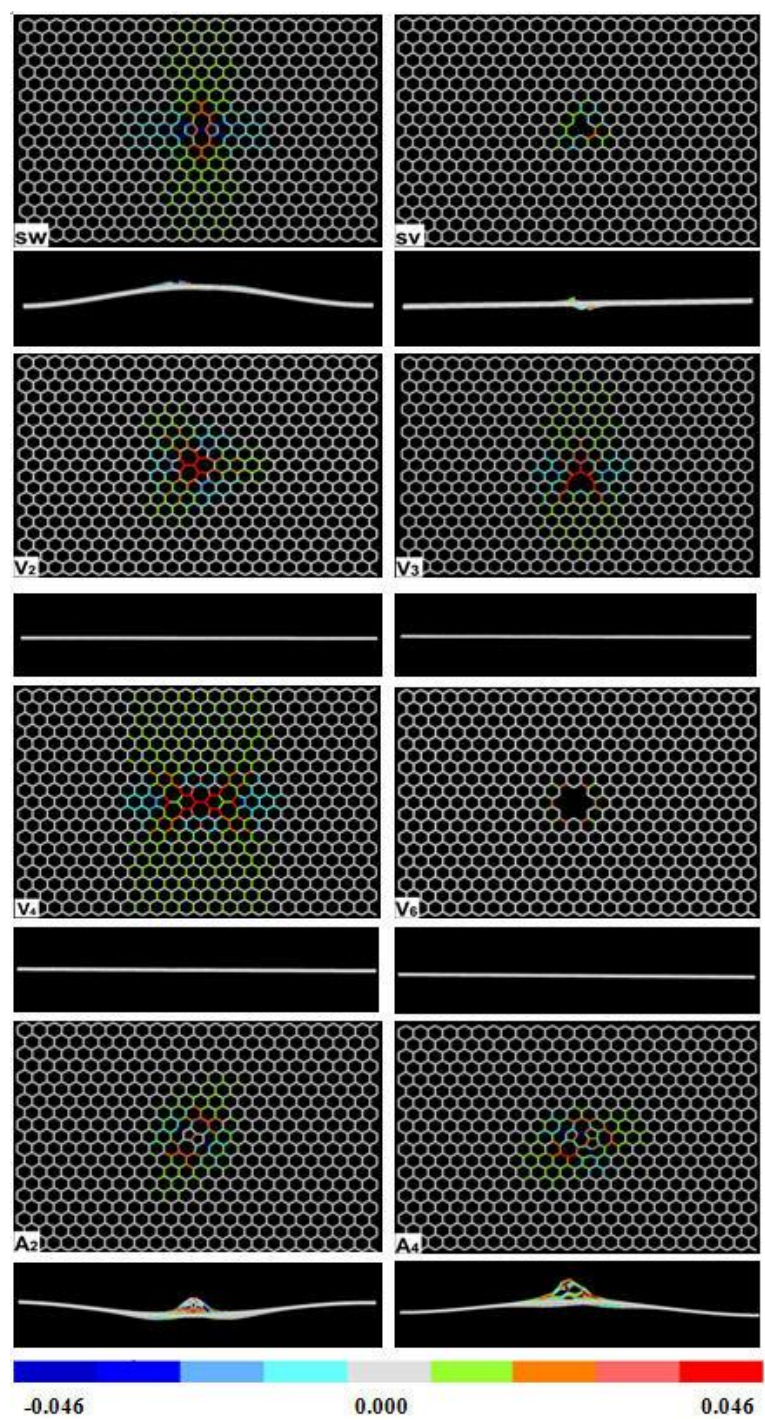

Fig. 2 Bond length distortion with respect to that of perfect graphene (1.42 $\AA$ ) induced by the defects. The magnitude and the sign of the distortions are indicated by colors as shown in the right bottom of the figure.

number of angles formed by nearest neighbor bonds; $\mathrm{N}_{d}$ is the number of dangling bond. $\mathrm{N}_{A 2}$ and $\mathrm{N}_{A 4}$ are the number of embedded atoms in $\mathrm{A}_{2}$ and $\mathrm{A}_{4}$ configurations, respectively. In this expression, the first two terms in the right hand side describe the 
elastic energy while the last three terms account for the electronic bond contribution to the defects energy. The coefficient $\alpha$ is the effective two-body force constant in graphene and $\quad \beta$ is related to the three-body force constant and measures the energy cost due to the bond angle distortions. The coefficient $\gamma$ gives the cost of electronic energy for creating a dangling bond, while $\delta_{2}$, and $\delta_{4}$ describe the electronic energy costs for adding an adatom in the $\mathrm{A}_{2}$ and $\mathrm{A}_{4}$ defects respectively. These coefficients are determined by fitting the energies from the TB calculations for the eight types of defects discussed above including those structures that have energies higher than their corresponding ground-state structure. We make $\delta_{2}$ different from $\delta_{4}$ based on the observation that the adsorption energy (per atom) of 2 adatoms on graphene is different from that of 4 adatoms on graphene due to the interaction among the adatoms.

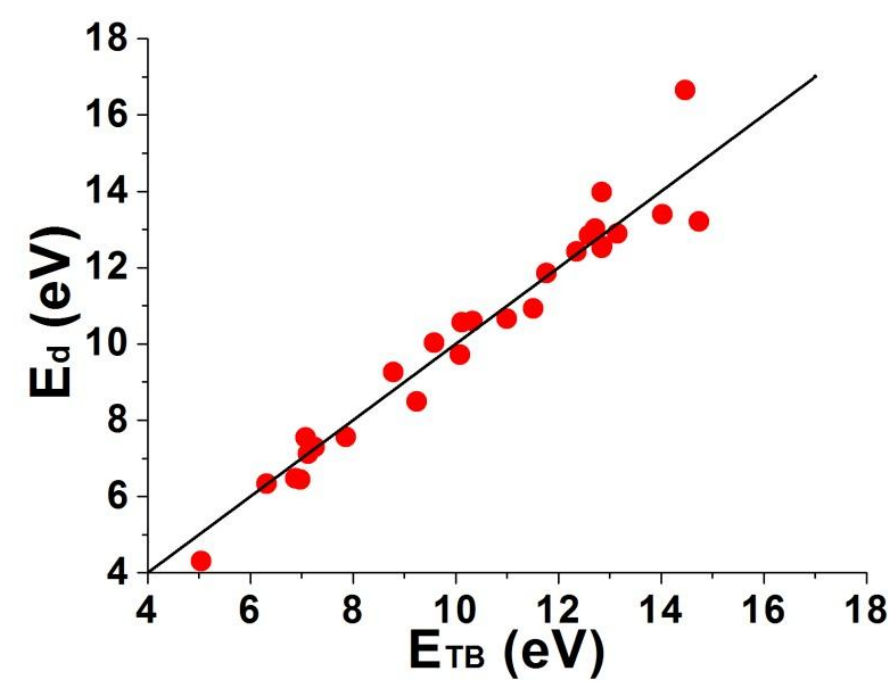

Fig. 3 Scatter plot of the defect formation energies obtained from the model (Eq. (1)) verses those from TB calculations. The black line $(y=x)$ indicates the perfect agreement between the two calculations. 
Fitting the energy expression of Eq. (1) to the defect energies from the TB calculations, the coefficients $\alpha, \beta, \gamma, \delta_{2}$, and $\delta_{4}$ are determined to be $9.227 \mathrm{eV} / \AA^{2}$, $11.594 \mathrm{eV}, 2.123 \mathrm{eV}, 1.682 \mathrm{eV}$, and $1.339 \mathrm{eV}$, respectively. Tang et al. showed the averaged formation energy of a dangling bond is about $2.3 \mathrm{eV}$ [30] which is close to the value obtained from our model. Using the parameters from the above fitting, the defect energies calculated the Eq. (1) are listed in Table I and compared with those from TB calculations. From the scatter plot of the energies obtained from the model (Eq. (1)) verses those from TB calculations as shown in Fig. 3, we can see that the model describes well the defect energies in graphene.

\subsection{Electron charge redistribution induced by defects}

We have also studied the electron charge redistribution caused by the defects. The electron charge on each atom is evaluated by Mulliken's charge analysis based on the eigenvectors of the Hamiltonian matrix and the elements of the overlap matrix from our tight-binding calculations. The Mulliken charge shared between orbital $\alpha$ of the $i^{\text {th }}$ atom orbital $\beta$ of the $j^{\text {th }}$ atom is defined as

$$
M_{i a, j \beta}=N^{-1} \sum_{\mathbf{k}} P_{a, \beta}^{i, j}(\mathbf{k}) S_{\beta, a}^{j, i}(\mathbf{k})
$$

with

$$
P_{a, \beta}^{i, j}(\mathbf{k})=\sum_{\mu} c_{\mu \mathbf{k}, i a} c_{\mu \mathbf{k}, j \beta}
$$

and

$$
S_{\beta, a}^{j, i}(\mathbf{k})=\left\langle\phi_{j, \beta} \mid \phi_{i, \alpha}\right\rangle e^{i \mathbf{k} \cdot \mathbf{R}_{n}}
$$

where $\mathbf{k}$ denotes the k-point and $\phi$ is the minimal basis orbital. In the present 
calculation, only $\Gamma$ point is used. The Mulliken charge shared between a pair of atoms can be obtained by summing over the relevant orbitals

$$
M_{i, j}=\sum_{\alpha \beta} M_{i \alpha, j \beta}
$$

And the gross atomic Mulliken charge can be expressed as

$$
M_{i}=\sum_{\alpha \beta j} M_{i \alpha, j \beta}
$$

The results of charge difference between defected and perfect graphene are shown in

Fig. 4. The deferential charge densities plotted in Fig. 4 are obtained by a Gaussian
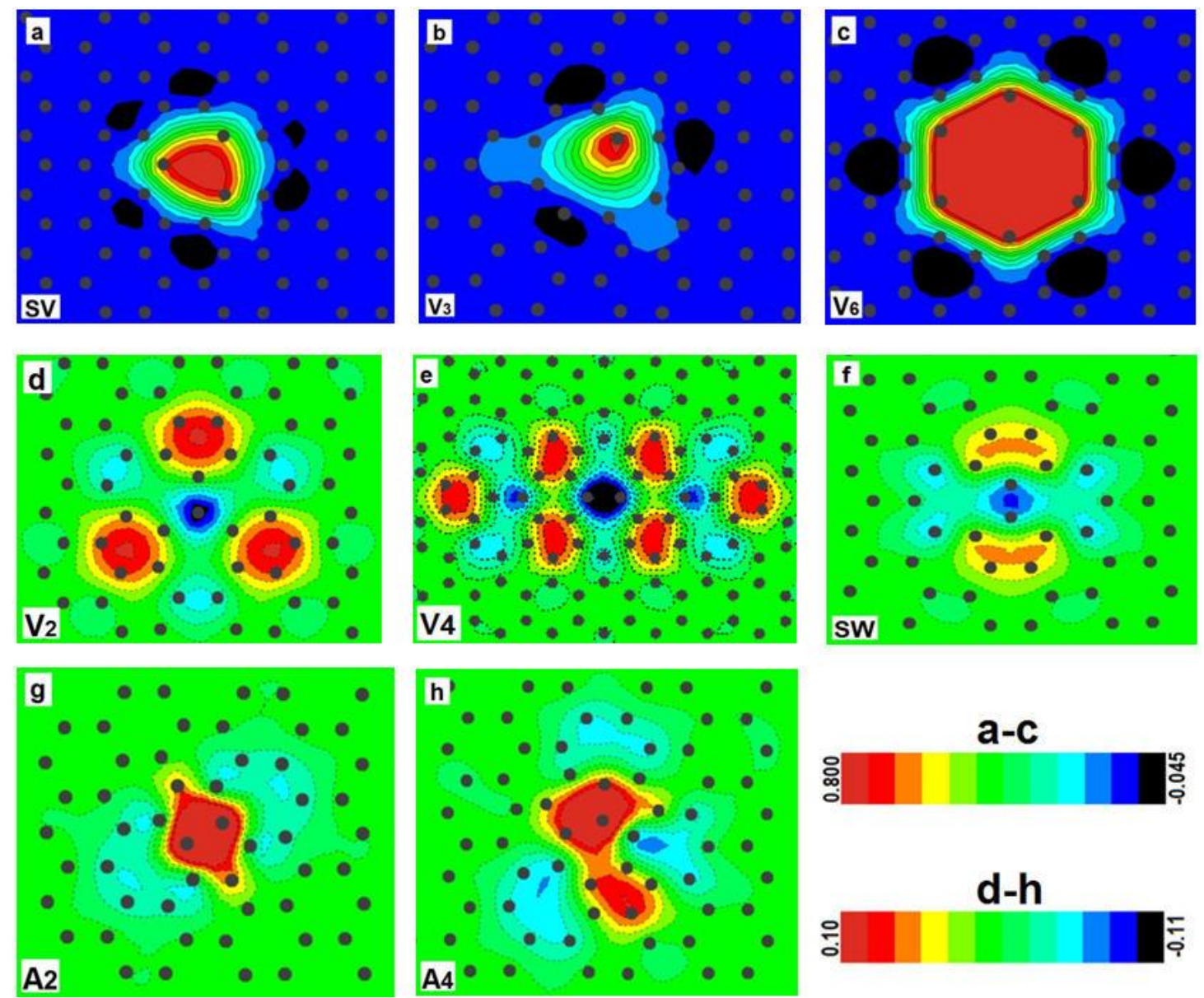

Fig. 4. The charge density difference is defect graphene relative to perfect graphene, including (a) SV(5-9), (b) $\mathrm{V}_{3}$ (5-10-5), (c) $\mathrm{V}_{6}$, (d) $\mathrm{V}_{2}$ (555-777), (e) $\mathrm{V}_{4}(5555-66-7777$ ), (f) $\mathrm{SW}(55-77)$, (g) $\mathrm{A}_{2}$ (7-55-7), and (h) $\mathrm{A}_{4}(55-6-5-777)$ defects. 
smearing factor of $0.001 \mathrm{e}$ on the Mulliken charge on each atom in defected and perfect graphene. Therefore, blue color indicates the loss of electrons and the red color indicates the gain of electrons on the carbon atoms induced by the defects. We found the charge difference is very prominent on the $\mathrm{SV}, \mathrm{V}_{3}$ and $\mathrm{V}_{6}$ defects as shown in Fig. 4 (a)-(c) where the charge varies from -0.045 to 0.8 e. This is because these defects exhibit dangling bonds (DB), 3 DB for SV, 1 DB for $\mathrm{V}_{3}$ and $6 \mathrm{DB}$ for $\mathrm{V}_{6}$. In comparison, the charge variation in $\mathrm{V}_{2}$ and $\mathrm{V}_{4}$ is much smaller as shown in Fig. 4 (d) and (e) where the charge variation in the plots is -0.11 to $0.1 \mathrm{e}$, because these defects do not have DBs. The charge variation in $A_{2}$ and $A_{4}$ defects is similar to that of $V_{2}$ and $\mathrm{V}_{4}$, while that of SW defect is the smallest among all the defects in this study. It is also interesting to note that pentagon defects tend to gain more electrons while heptagons loss electrons. The charge variation induced by the defects is well localized on the defects, and decay rapidly away from the defects.

\subsection{Defect induced electron localization}

In order to see the electron localization induced by the defect more clearly, we also calculated the electronic density-of-states (DOS) and the inverse participation ratio $\mathrm{P}^{-1}$ for each electronic state in the systems. The inverse participation ratio is defined for each eigenstate by $P^{-1}=\sum_{i=1}^{n}\left(c_{i}^{2}\right)^{2} /\left(\sum_{i=1}^{n} c_{i}^{2}\right)^{2}$ where $\mathbf{c}$ is the eigenvector of the corresponding eigenstate in the tight-binding minimal $\mathrm{sp}^{3}$ basis. By this definition, $\mathrm{P}^{-1}$ equals to 1 if the state is fully localized at one atom and $1 / \mathrm{N}$ if the state is uniformly spread over $\mathrm{N}$ atoms. Therefore $\mathrm{P}^{-1}$ can be used to quantitatively measure the 
localization of the state. As shown in Fig. 5, the $\mathrm{P}^{-1}$ for perfect graphene is very small and almost the same for every state (see Fig. 5a), indicating that all electronic states in graphene are delocalized as expected. On the other hand, defects in graphene are found to introduce some localized electronic states as signified by the large value of $\mathrm{P}^{-1}$, especially for the $\mathrm{SW}, \mathrm{V}_{1}, \mathrm{~V}_{6}, \mathrm{~A}_{2}$, and $\mathrm{A}_{4}$ defects. In comparison, $\mathrm{V}_{2}, \mathrm{~V}_{3}$ and
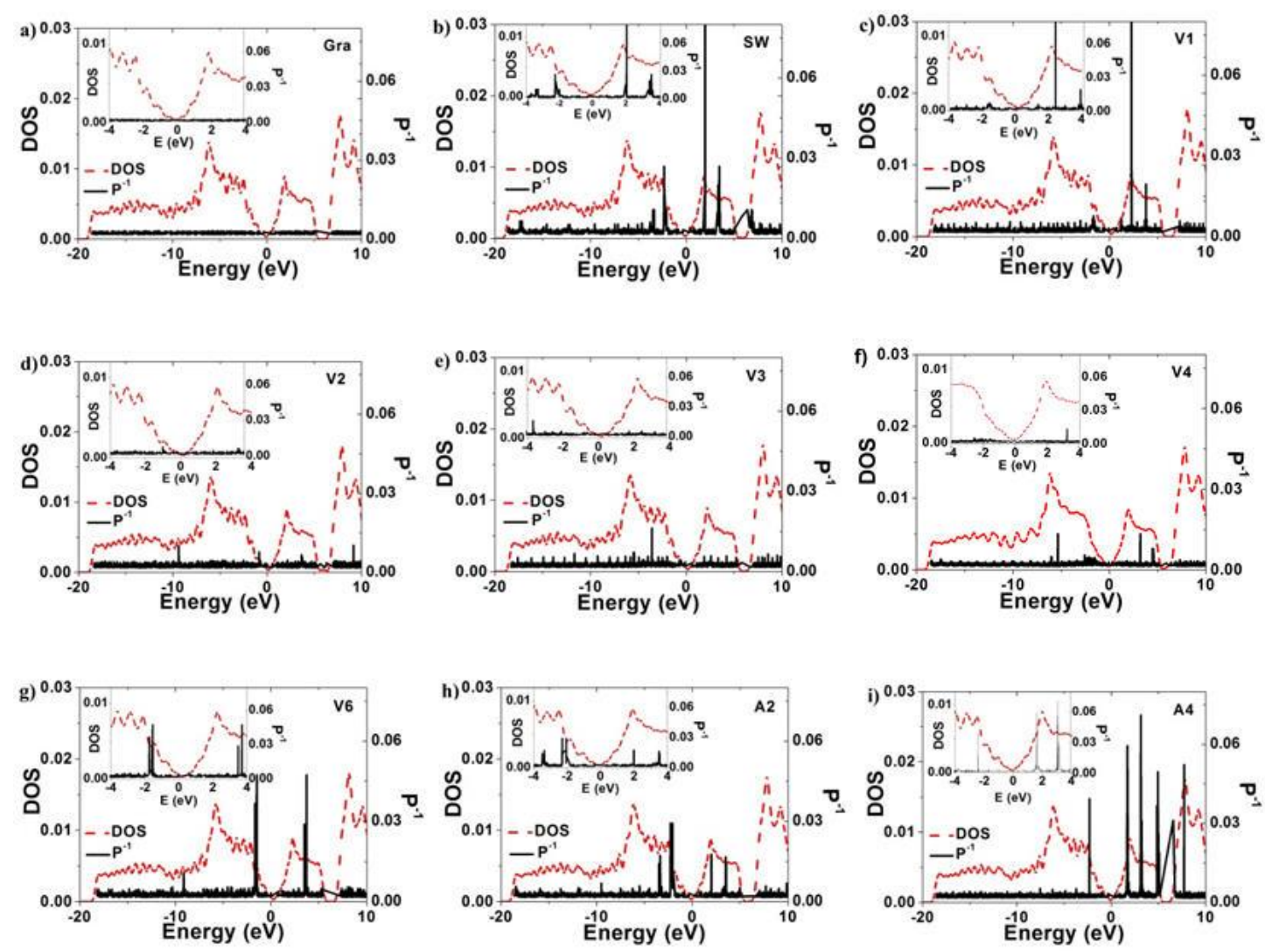

Fig. 5. The comparison of electronic density of states (Fermi level positioned at zero energy) and inverse participation ration of a) perfect graphene, b) $S W$, c) $V_{1}$, d) $V_{2}$, e) $V_{3}$, f) $V_{4}$, g) $V_{6}$, h) $A_{2}$, i) $\mathrm{A}_{4}$.

$\mathrm{V}_{4}$ defects introduce much less localized electronic states as one can from Fig. 5 (d) and (e). It is interesting to note all the localized electronic states introduced by the 
defects are located at least about $2 \mathrm{eV}$ away from the Fermi surface. This characteristic is different from the behavior of defect states in semiconductors where the localized defect states trend to lay in the energy close to the Fermi level. Localized defect state in graphene will cause extra electron scattering the affect the electron transport property on graphene. In this regard, the transport property is likely to be much less affected by the $V_{2} V_{3}$, and $V_{4}$ defects because the electronic states induced by these defects are much less localized.

\section{Conclusion}

In summary, we have studied the lattice distortion and electronic state localization caused by various point defects in graphene. We showed that the point defects can induce significant strain in graphene lattice at the location even far away from the center of the defect. The formation energy of the defect is well correlated with the bond-length variation and the bond-angle distortions caused by the defect, as well as the number of dangling bonds or embedded atoms in the defect. We also showed that these defects also introduce localized electronic states into graphene which would affect the electron transport properties of the graphene. 
- Author information

Corresponding Author

*E-mail: wangcz@ameslab.gov (CZ.W.); zhangw_bxx@jlu.edu.cn (W.Z.).

\section{Acknowledgments}

W. Zhang acknowledges the support by the National Natural Science Foundation of China (Grant No. 21173096), and supported by the State Key Development Program for Basic Research of China (Grant No.2013CB834801). Work at Ames Laboratory was supported by the US Department of Energy, Office of Science, Basic Energy Sciences, Division of Materials Science and Engineering, including a grant of computer time at the National Energy Research Supercomputing Centre (NERSC) in Berkeley, CA under Contract No. DE-AC02-07CH11358. 


\section{- REFERENCES}

[1] Novoselov KS, Geim AK, Morozov SV, Jiang D, Zhang Y, Dubonos SV, Grigorieva IV, Firsov AA. Science 2004; 306: 666.

[2] Novoselov KS, Geim AK, Morozov SV, Jiang D, Katsnelson MI, Grigorieva IV, Dubonos SV, Firsov AA. Nature 2005; 438: 197.

[3] Balandin AA, Ghosh S, Bao W, Calizo I, Teweldebrhan D, Miao F, Lau CN. Nano Lett. 2008; 8: 902.

[4] Choi SM, Jhi SH, and Son YW, Phys. Rev. B 2010; 81: 081407(R).

[5] Levy N, Burke SA, Meaker KL, Panlasigui M, Zettl A, Guinea F, Castro Neto AH, and Crommie MF. Science 2010; 329: 544.

[6] Lee C, Wei X, Kysar JW, and Hone J. Science 2008; 321: 385.

[7] Teague ML, Lai AP, Velasco J, Hughes CR, Beyer AD, Bockrath MW, Lau CN, and Yeh NC, Nano Lett. 2009; 9: 2542.

[8] Kim KS, Zhao Y, Jang H, Lee SY, Kim JM, Kim KS, Ahn JH, Kim P, Choi JY, and Hong BH. Nature 2009; 457: 706.

[9] Warner J H, Margine ER, Mukai M, Robertson AW, Giustino F, and Kirkland AI. Science 2012; 337: 209.

[10] Gui G, Li J, and Zhong J, Phys. Rev. B 2008; 78: 075435.

[11] Ni ZH, Yu T, Lu YH, Wang YY, Feng YP, and Shen ZX, ACS Nano 2008; 2: 2301.

[12] Craco L, Selli D, Seifert G, and Leoni S. Phys. Rev. B 2015; 91: 205120.

[13] Jain SK, Barkema GT, Mousseau N, Fang CM, and Marijn A. van Huis, J. Phys. Chem. C 2015; 119: 9646.

[14] Zhao QZ, Nardelli MB and Bernholc J, Phys. Rev. B 2002; 65: 144105.

[15] Jensen P, Gale J and Blase X. Phys. Rev. B 2002; 66: 193403.

[16] Li Z, Dharap P, Sharma P, Nagarajaiah S and Yakobson BI. J. Appl. Phys. 2005; 97 : 074303.

[17] Lehtinen O, Kurasch S, Krasheninnikov AVand Kaiser U. Nat. Commun. 2013; 4: 2089.

[18] Lee GD, Yoon E, He K, Robertson AW and Warner J H. Nanoscale 2014; 6: 14836.

[19] Warner JH, Lin YC, He K, Koshino M and Suenaga K. Nano Lett. 2014; 14: 6155.

[20] Kim K, Coh S, Kisielowski C, Crommie MF, Louie SG, Cohen ML and Zettl A. Nat. Commun. 2013; 4: 2723.

[21] Lee GD, Wang CZ, Yoon E, Hwang NM and Ho KM. Phys. Rev. B 2010; 81: 195419.

[22] Gong CC, He K, Robertson AW, Yoon E, Lee GD, and Warner JH. ACS Nano 2015; 656-662.

[23] Chen Q, Robertson AW, He K, Gong CC, Yoon E, Lee GD, and Warner JH. ACS nano 9 (2015) 8599-8608.

[24] Carpio A, Bonilla LL, Phys. Rev. B 2008; 78: 085406.

[25] Chen S, Chrzan DC. Phys. Rev. B 2011; 84: 214103.

[26] Grantab R, Shenoy VB, Ruoff RS. Science 2010; 330: 946.

[27] Lu WC, Wang CZ, Zhao LZ, Qin W, Ho KM. Phys. Rev. B 2015; 92: 035206.

[28] Lee GD, Wang CZ, Yoon E, Hwang NM, Kim DY and Ho KM. Phys. Rev. Lett. 95 (2005) 205501.

[29] Robertson AW, Lee GD, He K, Yoon E, Kirkland AI and Warner JH. Nano Lett. 2014; 14: 1634.

[30] Tang Z, Hasegawa M, Shimamura T, Nagai Y, Chiba T, Kawazoe Y, Takenaka M, Kuramoto E and Iwata T. Phys. Rev. Lett. 1999; 82: 12. 
Table 1. The bond-length and bond-angle distortions and defect energies $\mathrm{E}_{\mathrm{d}}$ using the information of bond-length and bond-angles distortions as well as the number of dangling bonds and embedded atoms from the expression of Eq. (1). The defect energies $\mathrm{E}_{\text {ТВ }}$ from the TB calculations are also shown for comparision.

\begin{tabular}{|c|c|c|c|c|c|c|c|}
\hline & $\Sigma(\Delta \mathrm{r})^{2}$ & $\Sigma\left(\Delta \theta / \theta_{\mathrm{o}}\right)^{2}$ & $\mathrm{~N}_{d}$ & $\mathrm{~N}_{A 2}$ & $\mathrm{~N}_{A 4}$ & $\mathrm{E}_{d}(\mathrm{eV})$ & $\mathrm{E}_{T B}(\mathrm{eV})$ \\
\hline $\mathrm{SW}$ & 0.053 & 0.329 & 0 & 0 & 0 & 4.30 & 5.05 \\
\hline $\mathrm{SV}$ & 0.003 & 0.004 & 3 & 0 & 0 & 6.45 & 6.97 \\
\hline $\mathrm{V}_{2}$ & 0.083 & 0.492 & 0 & 0 & 0 & 6.47 & 6.88 \\
\hline $\mathrm{V}_{3}$ & 0.281 & 0.507 & 1 & 0 & 0 & 10.59 & 10.33 \\
\hline $\mathrm{V}_{4}$ & 0.121 & 0.823 & 0 & 0 & 0 & 10.66 & 11.00 \\
\hline $\mathrm{V}_{6}$ & 0.007 & 0.018 & 6 & 0 & 0 & 13.02 & 12.02 \\
\hline $\mathrm{A}_{2}$ & 0.027 & 0.234 & 0 & 2 & 0 & 6.33 & 6.31 \\
\hline $\mathrm{A}_{4}$ & 0.033 & 0.310 & 0 & 0 & 4 & 9.26 & 8.79 \\
\hline
\end{tabular}

